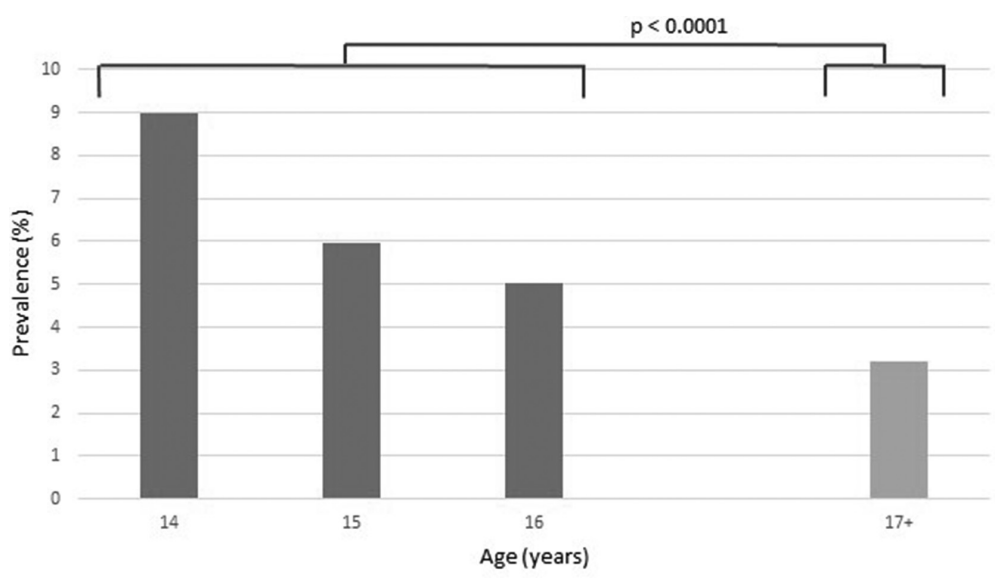

Abstract 29 Figure 1 Prevalence of short PR interval according to age

Abstract 29 Table 1 Table demonstrating the mean, standard deviations and p-values according to sex, ethnicity and age

\begin{tabular}{|l|l|l|l|}
\hline \multicolumn{2}{|l|}{ Variable } & Mean \pm SD & p value \\
\hline Sex & Male & $151.57+/-23.88$ & $<0.0005$ \\
\hline Female & $146.27+/-22.10$ & \\
\hline Ethnicity & White & $149.96+/-23.30$ & $<0.0005$ \\
\hline Age (years) & Non-White & $156.67+/-21.10$ & $<0.0005$ \\
\hline
\end{tabular}

enhanced resting AV node conduction. The high frequency of short PR in young athletes suggests that in the absence of an accessory pathway or symptoms its presence should not prompt further investigation. Long-term follow-up studies are required in order to draw definitive conclusions.

\section{THE PROGNOSTIC IMPLICATION OF TROPONIN LEVEL IN OVER 3000 PATIENTS PRESENTING WITH ATRIAL FIBRILLATION (NIHR HEALTH INFORMATICS COLLABORATIVE AF-TROP STUDY)}

${ }^{1}$ Amit Kaura* ${ }^{1}$ Ahran Arnold, 'Vasileios Panoulas, ${ }^{1}$ Ben Glampson, 2lim Davies, ${ }^{1}$ Abdulrahim Mulla, ${ }^{2}$ Kerrie Woods, ${ }^{3}$ Joe Omigie, ${ }^{4}$ Anoop D Shah, ${ }^{1}$ Keith Channon, 'Jonathan N Weber, 'Mark R Thursz, 'Paul Elliott, 'Harry Hemingway, 'Bryan Williams, ${ }^{1}$ Folkert Asselbergs, ${ }^{5}$ Michael O'Sullivan, ${ }^{6}$ Graham Lord, ${ }^{3}$ Narbeh Melikian, ${ }^{1}$ David Lefroy,

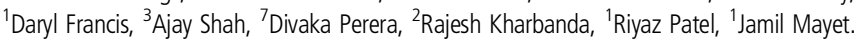
${ }^{1}$ NIHR Imperial College London Biomedical Research Centre and Imperial College Healthcare NHS Trust; ${ }^{2}$ NIHR University of Oxford BRC and Oxford University Hospitals NHS Foundation Trust; ${ }^{3}$ NIHR King's College London BRC and King's College Hospital NHS Foundation Trust; ${ }^{4}$ NIHR University College London BRC and University College London Hospitals NHS Foundation Trust; ${ }^{5}$ NIHR University of Cambridge BRC and Cambridge University Hospitals NHS Foundation Trust; ${ }^{6}$ NIHR King's College London BRC and Guy's and St Thomas' Hospital NHS Foundation Trust; ${ }^{7}$ King's College London

10.1136/heartjnl-2019-BCS.29

Background Patients presenting with atrial fibrillation (AF) often undergo measurement of troponin level, but interpretation of the result is impeded by uncertainty around its clinical importance. Although coronary artery disease (CAD) has been implicated in the prognostic significance of troponin levels in $\mathrm{AF}$, the role of investigating for CAD and its effect on mortality remain unclear. We investigated the relationship between troponin level, coronary angiography and all-cause mortality in real-world patients presenting with AF.

Methods We utilised National Institute for Health Research Health Informatics Collaborative data to identify patients admitted between 2010 and 2017 at 5 tertiary centres with a primary diagnosis of AF who underwent at least one troponin measurement. Patients meeting the eligibility criteria were followed up using routinely collected data, until death or censoring in April 2017. The peak troponin result during the index admission was scaled across assays as multiples of the upper limit of normal (xULN). To model non-linear relationships, we used restricted cubic splines for regression analyses.

Results 3,121 patients were included in the analysis. Over a median follow-up of 48.1 (IQR 30.5-64.9) months, there were $586(18.8 \%)$ deaths. While troponin levels below 1.3 $\mathrm{xULN}$ showed no significant relationship with hazard ratio, at higher troponin levels, a significant positive relationship was demonstrated (figure 1A). 216 (6.9\%) patients underwent coronary angiography, with 78 (36.1\%) of these patients subsequently undergoing coronary revascularisation. Above the ULN, there was a positive relationship between troponin level and odds of angiography (figure 1B).

While there was no significant relationship between troponin level and mortality in patients who underwent angiography (figure 1C), a significant relationship was observed in 
patients who did not undergo angiography with troponin levels above the ULN (figure 1D). On cox regression analysis, following adjustment for demographic and clinical factors, including troponin level, angiography was associated with a $39 \%$ reduction in mortality during follow-up (hazard ratio 0.61, 95\% CI 0.42-0.89, $\mathrm{p}=0.01$ ).

Conclusion Above the ULN, higher troponin levels were associated with higher mortality. Within the normal range, this was not the case. While angiography was not common in patients presenting with $\mathrm{AF}$, the likelihood of angiography increased at higher troponin levels. In cases where angiography was not performed, there was a direct relationship between troponin levels above the ULN and mortality; this was not evident where angiography was performed. Following adjustment for troponin level, angiography was associated with a significant reduction in mortality. The risk of mortality in patients presenting with AF may be mediated by CAD. Clinical trials are warranted to clarify the investigation and treatment of $\mathrm{CAD}$ in patients presenting with $\mathrm{AF}$ with elevated troponin levels.

Conflict of Interest No conflicts of interest

\section{UTILISATION OF AN EXISTING EXERCISE-BASED CARDIAC REHABILITATION PROGRAMME FOR PATIENTS WITH ATRIAL FIBRILLATION IN THE NATIONAL HEALTH SERVICE: PUTTING CURRENT SERVICES TO GOOD USE}

${ }^{1}$ Mark Mills*, ${ }^{2}$ Elizabeth Johnson, ${ }^{3}$ Hamza Zafar, ${ }^{4}$ Andrew Horwood, ${ }^{5}$ Nicola Lax, ${ }^{6}$ Sarah Charlesworth, ${ }^{7}$ Anna Gregory, ${ }^{8}$ Justin Lee, ${ }^{9}$ Jonathan Sahu, ${ }^{10}$ Graeme Kirkwood,

${ }^{11}$ Nicholas Kelland, ${ }^{12}$ Andreas Kyriacou. ${ }^{1}$ Sheffield Teaching Hospitals NHS Foundation Trust; ${ }^{2}$ Sheffield Teaching Hospital NHS Foundation Trust; ${ }^{3}$ Sheffield Teaching Hospital NHS Foundation Trust; ${ }^{4}$ Sheffield Teaching Hospital NHS Foundation Trust; ${ }^{5}$ Sheffield Teaching Hospital NHS Foundation Trust; ${ }^{6}$ Sheffield Teaching Hospital NHS Foundation Trust; ${ }^{7}$ Sheffield Teaching Hospital NHS Foundation Trust; ${ }^{8}$ Sheffield Teaching Hospital NHS Foundation Trust; ${ }^{9}$ Sheffield Teaching Hospital NHS Foundation Trust; ${ }^{10}$ Sheffield Teaching Hospital NHS Foundation Trust; ${ }^{11}$ Sheffield Teaching Hospital NHS Foundation Trust;

${ }^{12}$ Sheffield Teaching Hospital NHS Foundation Trust

\subsection{6/heartjnl-2019-BCS.30}

Introduction Exercise-based cardiac rehabilitation is an established intervention in the management of several cardiovascular conditions, including ischaemic heart disease and heart failure. There is increasing recognition for the role of exercise-based cardiac rehabilitation in the management of patients

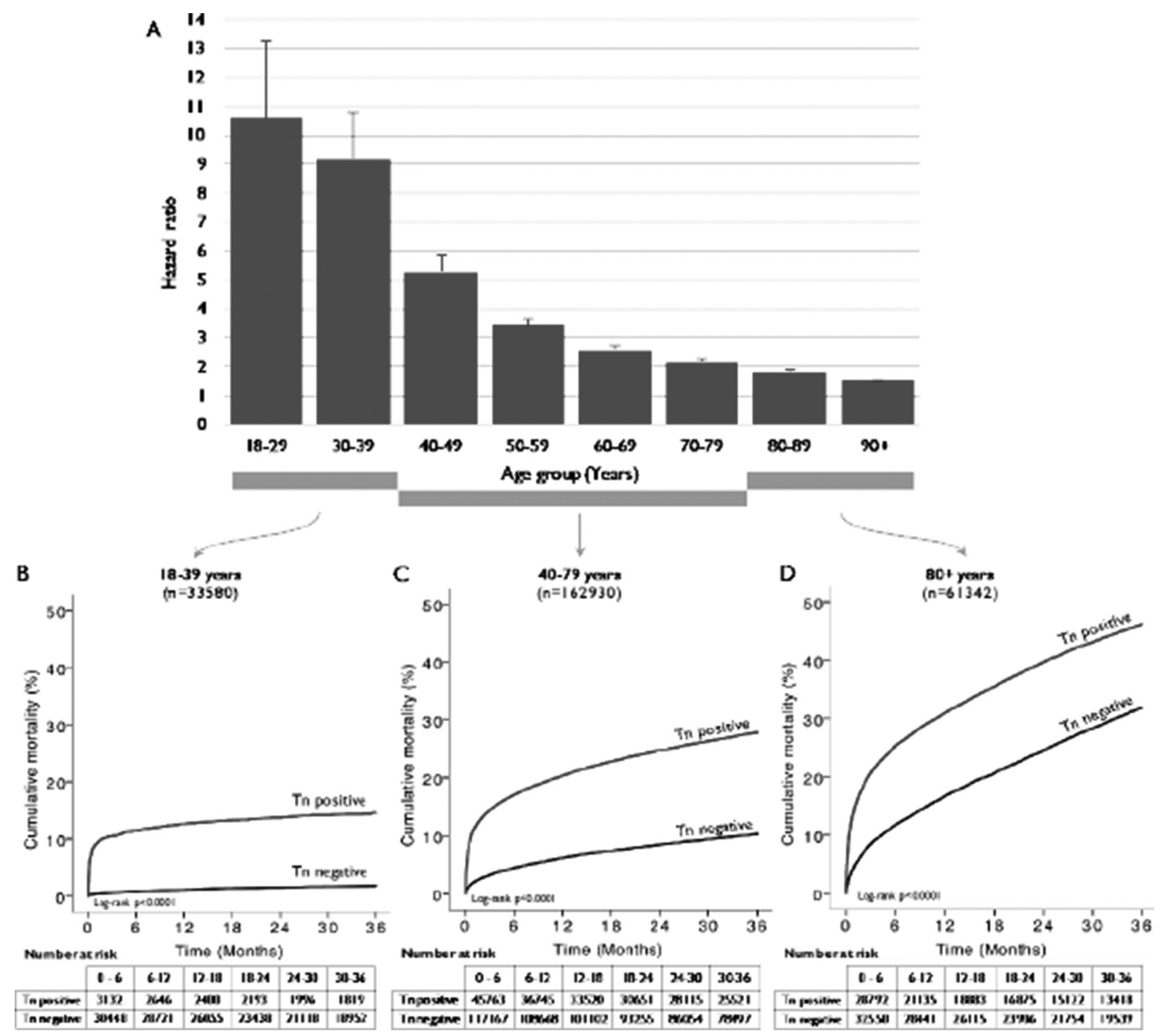

Abstract 30 Figure 1 A, Hazard ratios for troponin positive versus negative groups across different age bands for all patients; B-D, Kaplan-Meier survival curve by troponin positivity in 18-39, 40-79 and $80+$ year age bands.

Error bars denote upper $95 \%$ confidence interval. Tn, troponin. 